\title{
On the order of the recursion relation of Motzkin numbers of higher rank
}

\author{
Helmut Prodinger*
}

Mathematics Department, University of Stellenbosch, 7602 Stellenbosch, South Africa.

\begin{abstract}
For Motzkin paths with up- and down-steps of heights 1 and 2, the minimal recursion is of order 6, not of order 4, as conjectured by Schork.
\end{abstract}

Keywords. Motzkin numbers, recursion, gfun.

The classical Motzkin numbers count the numbers of Motzkin paths: We consider in the Cartesian plane $\mathbb{Z} \times \mathbb{Z}$ those lattice paths starting at $(0,0)$ that use an up-step $(1,1)$, a down-step $(1,-1)$, and a level-step $(1,0)$. Motzkin paths of length $n$ are built of these, lead to $(n, 0)$ and never go below the $x$-axis.

Now we consider higher rank Motzkin numbers, as suggested by Schork [2]: There are up-steps $(1,1)$, $(1,2), \ldots,(1, r)$ with respective weights $a_{1}, \ldots, a_{r}$ down-steps $(1,-1),(1,-2), \ldots,(1,-r)$ with respective weights $c_{1}, \ldots, c_{r}$, and a level-step $(1,0)$ with weight $b$.

Let us first consider the classical case $r=1$. The generating function $M(z)$ of these paths satisfies the equation

$$
M=1+b z M+a z M c z M,
$$

whence

$$
\frac{1-b z-\sqrt{1-2 b z+b^{2} z^{2}-4 a z^{2} c}}{2 a z^{2} c} .
$$

This equation is obtained by a decomposition of the Motzkin paths with respect to the first return to the $x$-axis.

Schork's first problem is to find a recursion for the numbers $m_{n}=\left[z^{n}\right] M(z)$. (The coefficient of $z^{n}$ in the power series expansion of $M(z)$, i.e., the number of (weighted) Motzkin paths of length $n$.)

This can be automatically solved with Maple's program gfun (written by Salvy et al.): The procedure algeqtodiffeq translates the (algebraic) equation for $M(z)$ into an equivalent differential equation:

$$
\begin{aligned}
2+(3 b z- & \left.b^{2} z^{2}+4 a z^{2} c-2\right) M \\
& +\left(-z+2 b z^{2}-z^{3} b^{2}+4 z^{3} a c\right) M^{\prime}=0 .
\end{aligned}
$$

The procedure diffeqtorec translates the differential equation into a recursion:

$$
\begin{aligned}
& \left(-b^{2}+4 a c\right)(n+1) m_{n} \\
& \quad+(5 b+2 b n) m_{n+1}-(n+4) m_{n+2}=0,
\end{aligned}
$$

which solves already this first problem. ${ }^{1}$

Now let us move to the instance $r=2$. Let us assume that the weights are all 1 , so that we are just interested to count the number of (generalized) Motzkin paths. In the paper [1] we find the equation for the generating function:

$$
\begin{aligned}
& z^{4} M^{4}-z^{2}(1+z) M^{3} \\
& +z(2+z) M^{2}-(1+z) M+1=0 .
\end{aligned}
$$

Thus (again with gfun)

$$
\begin{aligned}
-4-100 z^{2}+56 z \\
+\left(3750 z^{6}-5000 z^{5}+250 z^{4}\right. \\
\left.+700 z^{3}+160 z^{2}-92 z+4\right) M \\
+\left(-328 z^{2}+32 z-15250 z^{6}-20 z^{3}\right. \\
\left.+4750 z^{4}+11250 z^{7}-650 z^{5}\right) M^{\prime}
\end{aligned}
$$

\footnotetext{
*hproding@sun.ac .za

${ }^{1}$ After sending a draft of this note to M. Schork, he informed me that he could now also establish this recurrence together with Mansour and Sun.
} 


$$
\begin{aligned}
& +\left(5625 z^{8}-7750 z^{7}-1200 z^{6}+3880 z^{5}\right. \\
& \left.\quad-395 z^{4}-186 z^{3}+26 z^{2}\right) M^{\prime \prime} \\
& \quad+\left(625 z^{9}-875 z^{8}-250 z^{7}\right. \\
& \left.\quad+610 z^{6}-91 z^{5}-23 z^{4}+4 z^{3}\right) M^{\prime \prime \prime}=0
\end{aligned}
$$

and

$$
\begin{gathered}
625(n+3)(n+2)(n+1) m_{n} \\
-125(n+3)(n+2)(7 n+27) m_{n+1} \\
\quad-50(n+3)\left(5 n^{2}+24 n+23\right) m_{n+2} \\
+\left(41890+30860 n+7540 n^{2}+610 n^{3}\right) m_{n+3} \\
+\left(-6844-5151 n-1214 n^{2}-91 n^{3}\right) m_{n+4} \\
-(n+7)\left(23 n^{2}+301 n+976\right) m_{n+5}
\end{gathered}
$$

$$
+2(2 n+13)(n+8)(n+7) m_{n+6}=0 .
$$

(This recursion also appears in [1].)

Bruno Salvy has kindly informed me that this recursion of order 6 is minimal.

Schork [2] conjectured that there should be a $(2 r+1)$-term recursion (=order $2 r)$. Thus, the conjecture does not hold.

\section{REFERENCES}

[1] C. Banderier and P. Flajolet. Basic analytic combinatorics of directed lattice paths. Theoret. Comput. Sci., 281(12):37-80, 2002. Selected papers in honour of Maurice Nivat. [2] M. Schork. On the recursion relation of Motzkin numbers of higher rank. Online Journal of Analytic Combinatorics, 2:\#3, 2007. 